\title{
Technical Note: The heterogeneous Zeldovich factor
}

\author{
H. Vehkamäki, A. Määttänen, A. Lauri, I. Napari, and M. Kulmala \\ Department of Physical Sciences, Division of Atmospheric Sciences, University of Helsinki, Helsinki, Finland
}

\begin{abstract}
In this technical note we present the exact form of the Zeldovich factor for heterogeneous nucleation on spherical pre-existing particles. We study the error caused by planar pre-existing surface approximations, which have been used in our earlier heterogeneous nucleation model and elsewhere in the literature. We also test the significance of widely used approximations for cluster surface area and circumference. We conclude that the approximations do not affect the predicted onset saturation. Especially for small pre-existing particles the nucleation rates calculated with the exact and approximative models differ significantly.
\end{abstract}

\section{Introduction}

Formation of new aerosol particles via gas-to-particle conversion plays an important role in the atmosphere of Earth as well as that of planet Mars. In homogeneous nucleation molecular clusters are formed spontaneously from the vapour, whereas in heterogeneous nucleation clusters form on the surfaces of pre-existing particles, for example dust, thus facilitating the start of their condensational growth. Nucleation rate, or in the heterogeneous nucleation the nucleation probability, is the quantity accessible to laboratory and field measurements. The barrier for critical cluster formation, in other words the formation free energy, is the key quantity in predicting particle formation rate, and thus theorists focus on predicting it accurately. Nucleation rate is proportional to the exponential of the formation energy, and the kinetics of the cluster growth is described by two preexponential factors: the average growth rate of a critical cluster and the Zeldovich factor. The Zeldovich factor corrects among other things for the fact that some clusters that have reached the critical size still decay to smaller sizes. In one-component nucleation the homogeneous Zeldovich factor has a simple, well known analytical form, and an expression for the heterogeneous Zeldovich factor in the case of planar pre-existing surface has also been presented in the lit-

Correspondence to: H. Vehkamäki

(hanna.vehkamaki@helsinki.fi) erature. In this note we present a formula for the heterogeneous Zeldovich factor in the case where the pre-existing condensation nuclei are spherical.

\section{Theory}

In our previous article on one-component nucleation in the Martian atmosphere published in Journal of Geophysical Research - Planets (Määttänen et al., 2005) we derived a form of the one-component Zeldovich factor only applicable for a planar pre-existing condensation nucleus $(\mathrm{CN})$ surface. Pruppacher and Klett (Pruppacher and Klett, 1997, pp.202) state that the Zeldovich factor can be expressed as

$Z=\sqrt{\frac{\Delta \varphi^{*}}{3 \pi k T\left(N^{*}\right)^{2}}}$

for arbitrary geometries for which the surface area of the cluster, $A$, is proportional to $N^{2 / 3}$, where $N$ is the number of molecules in the cluster. $\Delta \varphi$ is the formation energy of the cluster, ${ }^{*}$ stands for the critical cluster, $T$ is the temperature and $k$ is the Boltzmann constant. Equation (11) in our paper Määttänen et al. (2005) is based on equation (1), and shows a relation between the homogeneous and heterogeneous Zeldovich factors. For a planar $\mathrm{CN}$ surface $A \propto N^{2 / 3}$, and the geometric factors $f=\Delta \varphi^{\text {het }} / \Delta \varphi^{\text {hom }}$ and $f_{n^{*}}=N^{\text {het }} / N^{\text {hom }}$ are equal, in which case relation (1) in fact simplifies into $Z_{\text {het }}=\frac{1}{\sqrt{f}} Z_{\text {hom }}$.

Here we derive the exact Zeldovich factor for onecomponent heterogeneous nucleation in the case of curved pre-existing surface; in this case the cluster surface area is not proportional to $N^{2 / 3}$. We also present some modelling results on its effect on nucleation. It should be noted that the formulation presented in Määttänen et al. (2005) is well applicable for large pre-existing particles acting as $\mathrm{CN}$, and thus the approximation does not affect the results of paper Määttänen et al. (2005), but for small CN the differences may be large. 


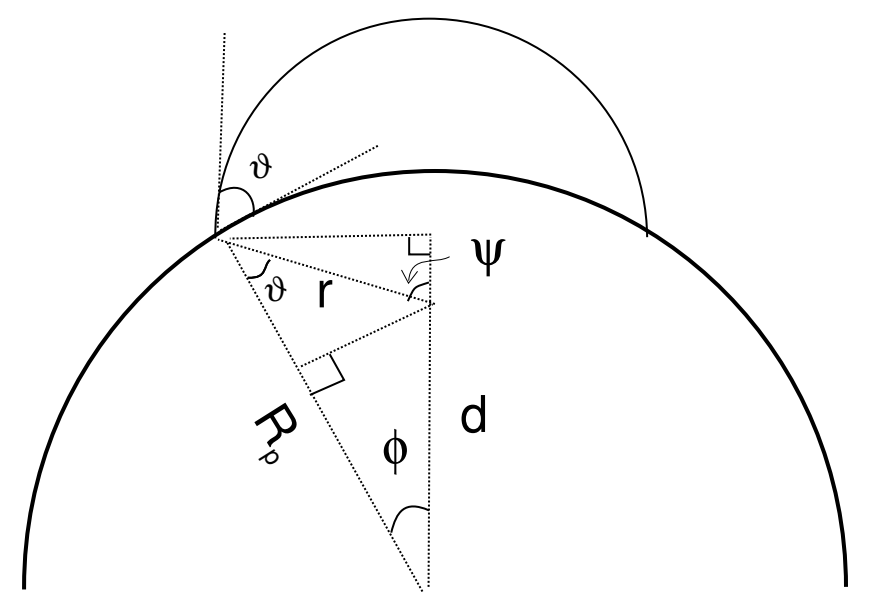

Fig. 1. The geometry of heterogeneous nucleation: a critical cluster of radius $r$ on the surface of a pre-existing particle of radius $\mathrm{R}_{p}$.

The Zeldovich factor is by definition

$Z_{\text {het }}=\sqrt{\frac{-1}{2 \pi k T}\left[\frac{\partial^{2} \Delta \varphi^{\text {het }}}{\partial\left(N^{\text {het }}\right)^{2}}\right]^{*}}$.

To obtain an explicit form for the Zeldovich factor we have to take the second derivative of formation free energy, which can be written

$$
\begin{aligned}
\Delta \varphi^{\text {het }}= & \Delta \varphi_{\mathrm{surf}}^{\mathrm{het}}+\Delta \varphi_{\mathrm{vol}}^{\mathrm{het}} \\
= & \sigma_{g, l} 2 \pi r^{2}(1-\cos \psi) \\
+ & \left(\sigma_{l, \mathrm{sol}}-\sigma_{g, \mathrm{sol}}\right) 2 \pi R_{p}^{2}(1-\cos \phi) \\
& +\Delta \mu N^{\text {het }}
\end{aligned}
$$

For simplicity of terminology we speak about a liquid cluster forming on a solid $\mathrm{CN}$, but the formulae are applicable also to the case of solid cluster forming on a solid CN. In Eq. (3) $\sigma_{g, l}$ is the gas-liquid surface tension, $\sigma_{l, \text { sol }}$ and $\sigma_{g \text {,sol }}$ are respectively the liquid-solid and gas-solid surface energies, $r$ is the radius of the cluster and $R_{p}$ the radius of the pre-existing particle. $\Delta \mu$ is the chemical potential difference between gas and liquid, both at the gas pressure. We have used the equimolar surface condition (Ono and Kondo, 1960; Laaksonen et al., 1999) for all the phase interfaces, which means that the total number of molecules in the cluster $N^{\text {het }}$ equals the number of molecules in the hypothetical bulk liquid since surface excess contributions are set to zero. The geometry of heterogeneous nucleation is shown in Fig. 1.

The angles $\phi$ and $\psi$ can be expressed as

$\cos \phi=\frac{R_{p}-r \cos \vartheta}{d}=\frac{X-m}{d_{X}}$

and

$\cos \psi=\frac{R_{p} \cos \phi-d}{r}=-\frac{r-R_{p} \cos \vartheta}{d}=\frac{-(1-X m)}{d_{X}}$ where

$d^{2}=r^{2}+R_{p}^{2}-2 r R_{p} \cos \vartheta=r^{2}\left(1+X^{2}-2 X m\right) \equiv r^{2} d_{X}^{2}$.

and $X=R_{p} / r$ and $m=\cos \vartheta$, where $\vartheta$ is the contact angle between the cluster and the pre-existing surface, as defined by Young's equation

$$
\cos \vartheta=m=\frac{\sigma_{l, \mathrm{sol}}-\sigma_{g, \mathrm{sol}}}{\sigma_{g, l}} .
$$

Now using (4), (5), (6) and (7) the surface term $\sigma_{g, l} 2 \pi r^{2}(1-$ $\cos \psi)+\left(\sigma_{l, \mathrm{sol}}-\sigma_{g, \mathrm{sol}}\right) 2 \pi R_{p}^{2}(1-\cos \phi)$ can be written as

$$
\begin{aligned}
\Delta \varphi_{\text {surf }}^{\text {het }}= & 2 \pi \sigma_{g, l}\left(r^{2}-m R_{p}^{2}\right. \\
& \left.+\frac{r^{3}-r^{2} R_{p} m-r R_{p}^{2} m^{2}+R_{p}^{3} m}{\sqrt{r^{2}-2 r R_{p} m+R_{p}^{2}}}\right) .
\end{aligned}
$$

We can easily calculate the first derivative of the volume term $\Delta \mu N^{\text {het }}$

$$
\frac{\partial \Delta \varphi_{\mathrm{vol}}^{\mathrm{het}}}{\partial N^{\text {het }}}=\Delta \mu .
$$

The first derivative of the surface term can be taken using the chain rule

$$
\begin{aligned}
\frac{\partial \Delta \varphi_{\text {surf }}^{\text {het }}}{\partial N^{\text {het }}} & =\left(\frac{\partial \Delta \varphi_{\text {surf }}^{\text {het }}}{\partial r}\right)\left(\frac{\partial r}{\partial N^{\text {het }}}\right) \\
& =\left(\frac{\partial \Delta \varphi_{\text {surf }}^{\text {het }}}{\partial r}\right)\left(\frac{\partial N^{\text {het }}}{\partial r}\right)^{-1} .
\end{aligned}
$$

From (10) it is seen that we also need the dependence of the number of molecules on the cluster radius, which can be acquired by expressing the heterogeneous cluster volume with the help of the geometry as

$$
\begin{aligned}
V^{\text {het }}= & \frac{\pi}{3} r^{3}\left(2-3 \cos \psi+\cos ^{3} \psi\right) \\
& -\frac{\pi}{3} R_{p}^{3}\left(2-3 \cos \phi+\cos ^{3} \phi\right) .
\end{aligned}
$$

We can express $N^{\text {het }}$ using the formulae (4), (5), (6) and (11), and the liquid phase molecular volume $v_{l}$, as

$$
\begin{aligned}
& N^{\text {het }}=\frac{V^{\text {het }}}{v_{l}}=\frac{\pi}{3 v_{l}}\left(2 r^{3}-2 R_{p}^{3}\right. \\
& \left.+\frac{2 r^{4}-2 r^{3} R_{p} m-r^{2} R_{p}^{2}\left(m^{2}-1\right)-2 r R_{p}^{3} m+2 R_{p}^{4}}{\sqrt{r^{2}-2 r R_{p} m+R_{p}^{2}}}\right) .
\end{aligned}
$$

Now we can calculate both derivatives needed in Eq. (10)

$$
\begin{aligned}
\frac{\partial \Delta \varphi_{\text {surf }}^{\text {het }}}{\partial r}= & 2 \pi r \sigma_{g, l} . \\
& \left(2+\frac{(1-m X)\left[2-4 m X-\left(m^{2}-3\right) X^{2}\right]}{\left(1-2 m X+X^{2}\right)^{3 / 2}}\right)
\end{aligned}
$$


and

$$
\begin{aligned}
\frac{\partial N^{\text {het }}}{\partial r}= & \frac{\pi r^{2}}{v_{l}} . \\
& \left\{2+\frac{(1-m X)\left[2-4 m X-\left(m^{2}-3\right) X^{2}\right]}{\left(1-2 m X+X^{2}\right)^{3 / 2}}\right\} .
\end{aligned}
$$

Combining the results (9), (14) and (15) we can see that the first derivative of the formation free energy is

$$
\begin{gathered}
\frac{\partial \Delta \varphi^{\text {het }}}{\partial N^{\text {het }}}=\frac{\partial \Delta \varphi_{\text {vol }}^{\text {het }}}{\partial N^{\text {het }}}+\frac{\partial \Delta \varphi_{\text {surf }}^{\text {het }}}{\partial r}\left(\frac{\partial N^{\text {het }}}{\partial r}\right)^{-1} \\
=\Delta \mu+\frac{2 \sigma_{g, l} v_{l}}{r} .
\end{gathered}
$$

This natural result explicitly confirms that (in the onecomponent case) setting the first derivative of the formation free energy to zero gives the Kelvin equation. The second derivative of the formation free energy thus becomes

$$
\begin{aligned}
& \left(\frac{\partial^{2} \Delta \varphi^{\text {het }}}{\partial\left(N^{\text {het }}\right)^{2}}\right)=\frac{-2 \sigma_{g, l} v_{l}}{r^{2}}\left(\frac{\partial r}{\partial N^{\text {het }}}\right) \\
& =\frac{-2 \sigma_{g, l} v_{l}}{r^{2}}\left(\frac{\partial N^{\text {het }}}{\partial r}\right)^{-1} \\
& =\frac{-2 \sigma_{g, l} v_{l}^{2}}{\pi r^{4}}\left[\frac{1}{2+\frac{(1-m X)\left[2-4 m X-\left(m^{2}-3\right) X^{2}\right]}{\left(1-2 m X+X^{2}\right)^{3 / 2}}}\right] .
\end{aligned}
$$

Inserting this result into (2) we can acquire the exact form of the Zeldovich factor

$$
\begin{aligned}
Z_{\mathrm{het}} & =\frac{v_{l}}{\pi r^{* 2}} \sqrt{\frac{\sigma_{g, l}}{k T}} \sqrt{\frac{1}{2+\frac{(1-m X)\left[2-4 m X-\left(m^{2}-3\right) X^{2}\right]}{\left(1-2 m X+X^{2}\right)^{3 / 2}}}} \\
& =Z_{\mathrm{hom}} \sqrt{\frac{4}{2+\frac{(1-m X)\left[2-4 m X-\left(m^{2}-3\right) X^{2}\right]}{\left(1-2 m X+X^{2}\right)^{3 / 2}}}} .
\end{aligned}
$$

It should be noted that the heterogeneous Zeldovich factor $Z_{\text {het }}$ reduces to the homogeneous one $Z_{\text {hom }}=v_{l} /\left(2 \pi r^{* 2}\right) \sqrt{\sigma_{g, l} /(k T)}$ in the case when $m=-1(\mathrm{CN}$ surface completely non-wetting) or $X=0$ (radius of the $\mathrm{CN}$ approaches zero), as it should.

The paper Määttänen et al. (2005) included also two other approximations. The cluster circumference was approximated in the nucleation rate expressions (8) and (12) of reference Määttänen et al. (2005) as $\pi r \sin \vartheta$, which is again an approximation for a planar pre-existing surface. The correct form for a spherical pre-existing particle is $2 \pi R_{p} \sin \phi$, where $\sin \phi$ can be calculated with the help of Eq. (4).

The direct vapour desorption approach for the growth rate of the embryo was presented in the comparison of the models. The formula (14) in Määttänen et al. (2005) includes an

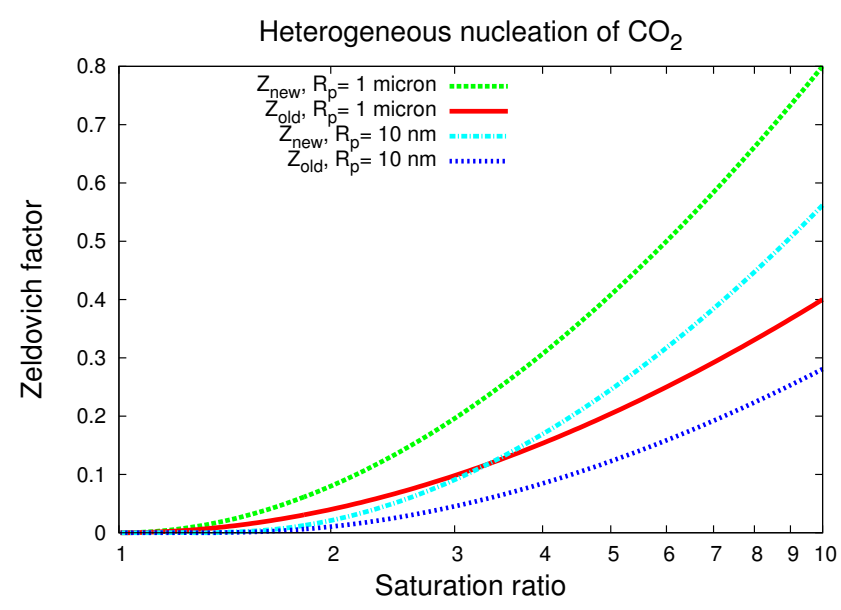

Fig. 2. The exact (long-dashed line for $1 \mu \mathrm{m}$ and dotted line for $10 \mathrm{~nm}$ ) and the approximative (solid line for $1 \mu \mathrm{m}$ and short-dashed line for $10 \mathrm{~nm}$ ) Zeldovich factors as a function of saturation ratio.

approximation also mentioned in the text: the cluster cap surface area has been approximated as $\pi r^{2}$. The real cap surface area can be easily calculated as

$A_{\text {het }}=2 \pi r^{2}\left(1+\frac{r-R_{p} \cos \vartheta}{d}\right)$.

\section{Model results}

We conducted some of the model runs described in paper Määttänen et al. (2005) again with the exact formulations included in the model. Here we present results for $\mathrm{CO}_{2} \mathrm{nu}$ cleation only. The model runs were made in the Martian atmospheric conditions with $95.32 \% \mathrm{CO}_{2}$ atmosphere, and the temperature range in the runs was $100-150 \mathrm{~K}$. The preexisting particle radius $R_{p}$ was $1 \mu \mathrm{m}$ which is near the mean radius of Martian dust near the surface. Here some runs were made also using a radius $R_{p}$ of $10 \mathrm{~nm}$ to test the effect of the planar surface approximations for small pre-existing particles.

Figure 2 shows that the exact Zeldovich factors are somewhat larger than the approximations for a planar surface, and the difference grows when the critical cluster gets smaller with increasing saturation ratio $S$. It can also be seen that the difference is smallest at the saturation ratios between 1 and 2 where nucleation onset happens, and thus the conclusion is that the inaccurate Zeldovich factor does not affect the prediction of nucleation onset significantly.

Figure 3 shows the nucleation rate $J$ and the nucleation probability $P$ calculated for $1 \mu \mathrm{m}$ pre-existing particles with both the exact and the approximative models. The nucleation rates differ very little, and the nucleation probabilities reach values near unity at the same saturation ratio. The difference in nucleation probabilities is seen only for very small values 


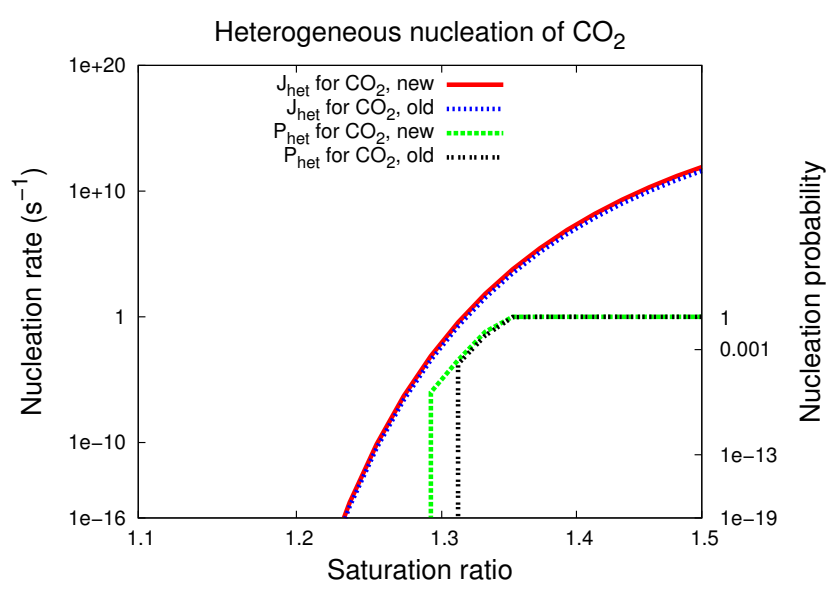

Fig. 3. The nucleation rates (solid line for the exact and shortdashed line for the approximations) and the nucleation probabilities (long-dashed line for the exact and dotted line for the approximation) as a function of saturation ratio.

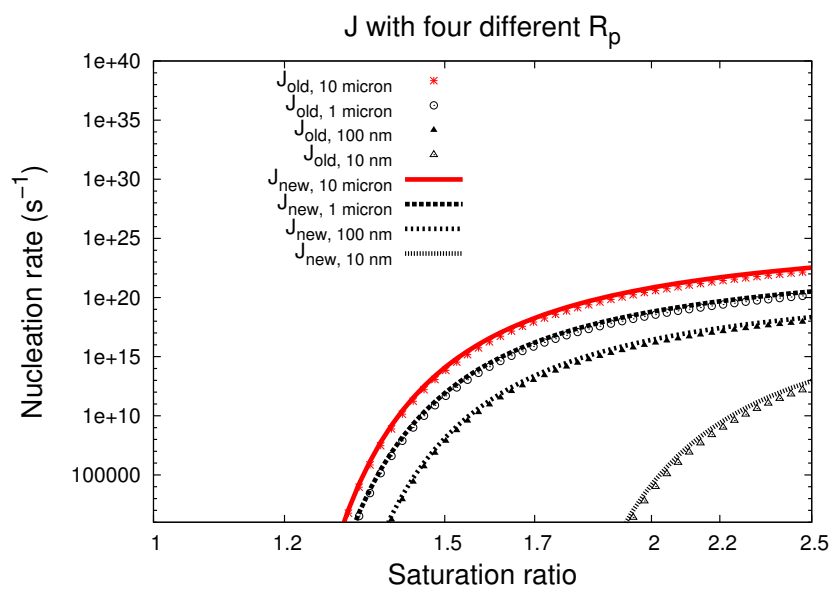

Fig. 4. The nucleation rates as a function of saturation ratio for four different $R_{p}$ sizes, $10 \mathrm{~nm}, 100 \mathrm{~nm}, 1 \mu \mathrm{m}$ and $10 \mu \mathrm{m}$.

of $P$ (note the logarithmic scale). The onset ( $P$ exceeds 0.5 ) happens at the same values of $S$ for both models.

Figure 4 shows the nucleation rate as a function of the saturation ratio for four different $\mathrm{CN}$ radii. The difference between the exact and approximative formulations is small, but visible, and especially so for small pre-existing particles.

Figure 5 shows the difference in the cap surface areas calculated with the approximation $\pi r^{2}$ and the exact formulation given by Eq. (18). The difference increases with decreasing saturation ratios, which is natural, since the critical cluster is largest with small values of $S$ and thus the error in the cap surface area is also largest. It can be seen that for different pre-existing particle sizes the behaviour of the

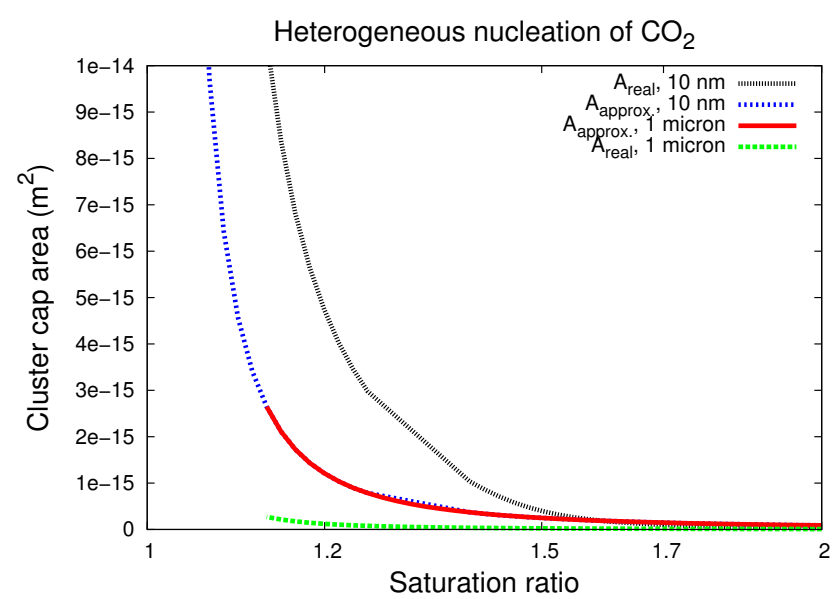

Fig. 5. The exact (dotted line for $10 \mathrm{~nm}$ and long-dashed line for $1 \mu \mathrm{m}$ ) and the approximative (short-dashed line for $10 \mathrm{~nm}$ and solid line for $1 \mu \mathrm{m}$ ) cluster cap surface areas as a function of saturation ratio.

error is different. The plot shows that for $10 \mathrm{~nm}$ and $1 \mu \mathrm{m}$ pre-existing particles the approximated cap surface areas are equal, as it should since the approximate area does not depend on the pre-existing particle size. With the exact approach the influence of the pre-existing particle size on the cap surface area is clearly seen.

\section{Conclusions}

The theory of heterogeneous nucleation is not complete without a correct form for the Zeldovich factor. We present an analytical expression for the Zeldovich factor in onecomponent heterogeneous nucleation with spherical condensation nuclei. This result absolves us from the unnecessary approximation of using the homogeneous Zeldovich factor, or the heterogeneous factor for planar pre-existing surface, when the condensation nuclei are small. We tested the difference in numerical results obtained using approximate and accurate Zeldovich factor in a case of $\mathrm{CO}_{2}$ nucleation in Martian atmosphere described in our earlier paper Määttänen et al. (2005). The exact Zeldovich factor give larger nucleation rates than the approximate, but the difference is less than one order of magnitude. The difference is smallest between saturation ratios one and two, and thus the onset of nucleation is well predicted even with the approximate Zeldovich factor. As expected, the inaccuracy of the approximate method increases when the size of the condensation nuclei decreases, but it is clearly detectable even with nuclei of one micron radius.

Acknowledgements. We thank the Academy of Finland and the Kordelin foundation for funding. 


\section{Edited by: T. Koop}

\section{References}

Laaksonen, A., McGraw, R., and Vehkamäki, H.: Liquid-drop formalism and free-energy surfaces in binary homogeneous nucleation theory, J. Chem. Phys., 111, 2019-2027, 1999.
Määttänen, A., Vehkamäki, H., Lauri, A., Merikallio, S., Kauhanen, J., Savijärvi, H., and Kulmala, M.: Nucleation studies in the Martian atmosphere, J. Geophys. Res., 110, E02002, doi:10.1029/2004JE002308, 2005.

Ono, S. and Kondo, S.: Encyclopedia of Physics, p. 134, Springer, Berlin, 1960.

Pruppacher, H. R. and Klett, J. D.: Microphysics of Clouds and Precipitation, Kluwer Acad., Norwell, Mass., 1997. 